\title{
Welche Patienten profitieren?
}

- Trotz der Kriterien zur Differenzialdiagnostik von Asthma bronchiale und COPD stellt sich im klinischen Alltag oft die Frage, in welche Kategorie ein Patient einzuordnen ist. „Das Problem ist, dass wir in klinischen Studien Phänotypen untersuchen, die wir im klinischen Alltag nicht sehen", betonte Prof. Winfried Randerath, Solingen.

\section{Differenzialdiagnose erschwert}

Zwischen Asthma und COPD gibt es in der Praxis viele Überschneidungen. Das gilt besonders für das schwere oder refraktäre Asthma, das durch zunehmende chronische Inflammation und Obstruktion gekennzeichnet ist, deren Reversibilität immer mehr verloren geht. Häufig sind dann nicht nur größere, sondern auch kleinere Atemwege involviert. In der Computertomografie sieht man eine Zunahme der Wanddicke der Bronchien wie beim Bronchitistyp der COPD. So wird es immer schwieriger, COPD und Asthma zu unterscheiden. Beim schweren Asthma etwa ist das Lumen der Bronchien durch die Wandverdickung wie bei der COPD vermindert.

\section{Leukotriene triggern Entzündung}

Die COPD wird grob in Emphysemtyp und Atemwegstyp eingeteilt. Je nach Schweregrad ist aber auch mehr oder weniger Entzündung im Spiel. Dabei überwiegen Neutrophile und Makrophagen, während beim Asthma die eosinophile Entzündung dominiert. In beiden Fällen sind auch Leukotriene involviert.

\section{ACT-Score verbessert}

Wie Prof. Christian Virchow, Rostock, erklärte, kann der Leukotrienrezeptorantagonist Montelukast (Singulair ${ }^{\circledast}$ ) die Kontrolle eines persistierenden Asthma bronchiale verbessern, wenn dies durch inhalative Steroide und lang wirksame Betamimetika nicht ausreichend möglich ist. Dies hat die MONICAStudie erneut bestätigt [1]. Die zusätzliche antientzündliche Therapie führte zu einer signifikanten und anhaltenden Verbesserung

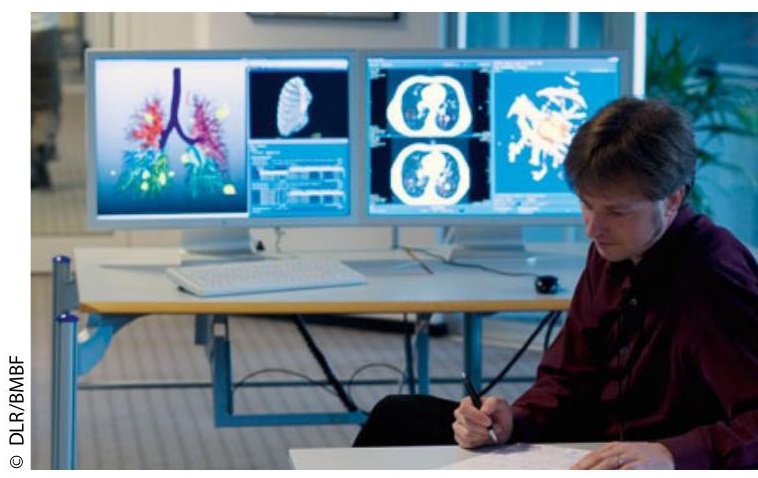

Häufig sind bei schwerem Asthma auch die kleineren Atemwege betroffen.

des Scores im Asthma-control-test (ACT). Besonders deutlich profitierten gemäß einer Subgruppenanalyse Patienten mit niedrigen ACT-Ausgangswerten, mit gleichzeitiger allergischer Rhinitis, jüngere Patienten mit kürzerer Asthmaanamnese und Patienten, die nur mit Steroiden behandelt wurden.

$a b$

1.Virchow JC et al. Respir Med 2010;104:644-651

Symposium „Asthma \& COPD“ anlässlich des 52. DGP-Kongresses am 9. April 2011 in Dresden (Veranstalter: MSD Sharp \& Dohme)

\section{Neue Therapieoption bei NSCLC}

\section{Tyrosinkinase irreversibel und effektiv blockiert}

\begin{abstract}
- Der Mutationsstatus des epidermalen Wachstumsfaktorrezeptors (EGFR) erlaubt eine zielgerichtete, individuelle Therapie des nicht kleinzelligen Lungenkarzinoms (NSCLC) mit Tyrosinkinaseinhibitoren (TKI). Bei 50-70\% der Patienten mit aktivierender EGFR-Mutation kommt es jedoch vor oder unter der Behandlung mit den EGFR-Inhibitoren zu sekundären Resistenzmutationen - etwa der T790MMutation - und in der Folge zu Rezidiven. Eine neue Option für Patienten mit Progress nach TKI-Versagen ist Afatinib (BIBW 2992, Tomtovok $^{\mathrm{TM}}$ ). Im Gegensatz zu bisher zugelassenen TKI blockiert der orale TKI selektiv und irreversibel zwei erbB-Rezeptor-Tyrosinkinasen: EGFR (erbB1) und HER2 (erbB2).
\end{abstract}

\section{Progressionsfreies Überleben}

Derzeit wird Afatinib bei verschiedenen soliden Tumoren klinisch erprobt. Phase II- und IIIDaten des LUX-Lung-Studienprogramms zum NSCLC stellte Prof. Dr. Rainer Wiewrodt, Münster, vor. In LUX-Lung 1 wurden 585 Patienten mit fortgeschrittenem NSCLC und Progress nach ein oder zwei Chemotherapien und anschließender TKI-Behandlung (Erlotinib oder Gefitinib) mit Afatinib oder Placebo jeweils in Kombination mit Best Supportive Care behandelt [1]. Ein Überlebensvorteil konnte in der Phase IIb/III-Studie nicht gezeigt werden. „Die Mehrheit der Patienten sprach aber in dieser Dritt- und Viertbehandlungssituation an", erklärte Wiewrodt. Die Tumorkontrollrate erreichte 58\% (Placebo: 19\%). Das progressionsfreie Überleben (PFS) verlängerte sich von 1,1 auf 3,3 Monate. In einer Subgruppenanalyse von Patienten, die nach klinischen Kriterien wahrscheinlich einen mutierten EGFR hatten, zeigte sich ein PFS von 4,4 Monaten und einen Trend in Richtung verlängertes Gesamtüberleben (OS).

\section{Tumoren deutlich verkleinert}

In LUX-Lung 2 erhielten 129 Patienten mit fortgeschrittenem NSCLC und EGFR-Mutation, die chemonaiv oder nach Erstlinien- therapie progredient waren, den TKI [2]., In der Phase II-Studie zeigten sich hohe Ansprechraten mit deutlicher Verkleinerung der Tumoren", berichtete Wiewrodt. Das mediane OS wurde in der Gruppe der ersttherapierten Patienten bislang nicht erreicht, für die Zweitlinientherapie war es 24 Monate. Das mediane PFS war für Patienten mit aktivierender EGFR-Mutation 15 Monate (del 19/L858R). Hinsichtlich des Sicherheitsprofils unterscheidet sich Afatinib nicht von anderen TKI. Typische unerwünschte Wirkungen wie Durchfall und Hautausschlag seien mit Dosisanpassung und supportiver Therapie gut beherrschbar, resümierte Wiewrodt. koc

1. Miller VA et al. ESMO 2010. abstract ID: LBA1 2. Yang $C$ et al. ESMO 2010. abstract: 367PD

Pressegespräch „Die Therapie des Lungenkrebs im Wandel" anlässlich des 52. DGPKongresses am 7. April 2011 in Dresden (Veranstalter: Boehringer Ingelheim) 